\title{
EXAMINING A DEEP LEARNING NETWORK SYSTEM FOR IMAGE IDENTIFICATION AND CLASSIFICATION FOR PREVENTING UNAUTHORIZED ACCESS FOR A SMART HOME SECURITY SYSTEM
}

\author{
Beloved Egbedion, Georgia Southern University, be02054@georgiasouthern.edu \\ Hayden Wimmer, Georgia Southern University,hwimmer@georgiasouthern.edu \\ Carl M. Rebman Jr., University of San Diego, carlr@sandiego.edu \\ Loreen M. Powell, Bloomsburg University, lpowell@bloomu.edu
}

\begin{abstract}
There are many different smart home surveillance and control systems, which will need some type of visual identification and classification system. Past models of Deep Learning have had great success in visual identification and image classification particularly in the healthcare and security industries. This study reviews past architecture and applications of Deep Learning and Convolutional Neural Networks. This paper then presents the creation, process, testing, and results of a CNN model with the end objective of identifying images for determination of access rights. Evaluation outcomes show that after 50 forward and backward dataset training passes the deep learning network achieved an identification accuracy of $96.7 \%$ and a $98.0 \%$ probability of proper classification of access authorization. The results suggest that deep learning models could be successful in strengthening smart home security systems.
\end{abstract}

Keywords: Deep Learning, Convolutional Neural Networks, Image Classification, Smart Home, Security

\section{INTRODUCTION}

Americas are increasingly become more concerned about home security For example, in a study by asecurelife.com $63 \%$ percent of Americans between the ages of 25 and 34 are more concerned about a home invasion than financial security and 53\% more women feared home invasion over identity theft. Advances in smart home technologies are helping to address these fears and concerns. According to the International Energy Agency (IEA 2013) the smart home market is projected to be $\$ 26$ billion in 2019 (up from $\$ 40$ million in 2012). This forecast is echoed by Statista, which also predicts the smart home market to be $\$ 27$ billion and $\$ 44.7$ billion in 2023 (Statista 2018).

There are also many risks in smart home security systems (Fernandes et al 2016). For example, smart home security systems have to be prepared to address cyberattacks that would allow criminals to monitor, steal personal information, or lock the person out of their home. According to Cate Lawrence of readwrite.com, there are three main layers of cyber defense (Lawrence, 2017). The first or perimeter layer is about preventing unauthorized access of one's devices. The second layer is about intrusion detection and prevention. The third is anomaly detection and behavioral analysis. An area that has been used to assist with these three layers of cyber defense is Deep learning.

Deep learning, a popular machine learning technique being used to establish state of the art solution for problems that ranges from Natural Language Processing (NLP) to object classification. Deep learning has recently seen rapid development and received significant attention due to its state-of-the-art performance on previously thought hard problems (Hohman et al 2017). In the last five years, the deep learning technique has achieved superior results over other classical machine learning methods in image classification, detection and segmentation in several applications (Ghazi et al 2017; Hinton et al 2012; Le et al 2012; Shu et al 2017; Yu et al 2017).

Deep learning and neural networks are terms that many people use interchangeably and yet there is a difference between the two. They are both are techniques that exist under the global umbrella of artificial intelligence along with machine learning. According to Skymind (2019), it is best to consider all the techniques "to be like a set of Russian dolls nested within each other, beginning with the smallest and working out." In other words, Deep learning is a subset of machine learning, and machine learning is a subset of AI (Dhande 2018)." Deep learning is also referred to 
as deep neural networks (Bahmani, 2018). Figure 1 illustrates the differences between the artificial intelligence, machine learning and deep learning.

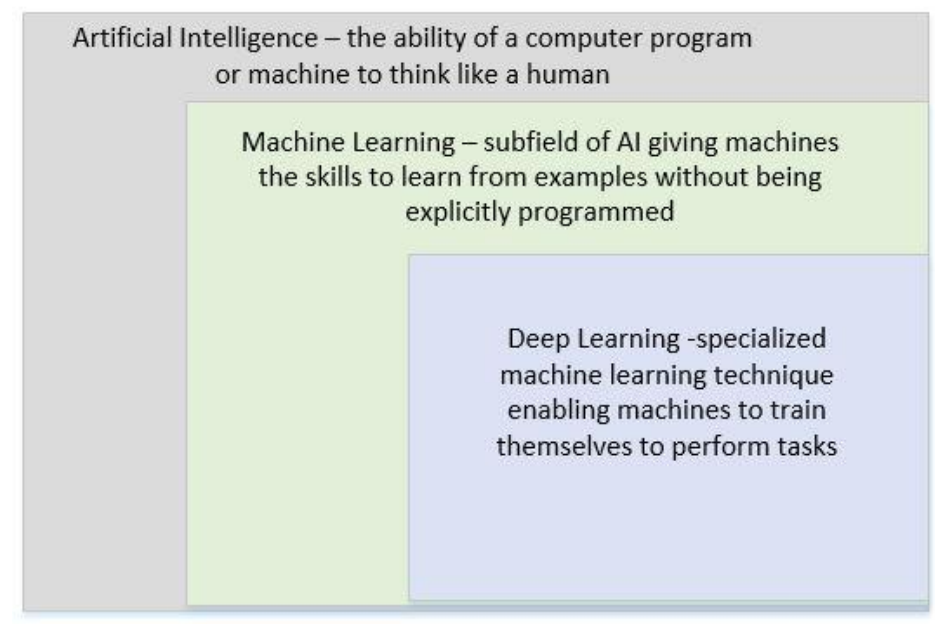

Figure 1. Differences between AI, ML, and DL.

The format of this study is as follows. First is a discussion of a relevant literature followed by methodology discussion and test results. Included with the methodology is detailed discussion of data collection and execution of the CNN model and development. The manuscript concludes with results, limitations, and future research.

\section{LITERATURE REVIEW}

While the task of categorizing images or Image Classification is second nature for humans, it presents a challenge for computers. Computers have difficulty in analyzing the variability in object types, classification challenge, non-linear processing, and accuracy in feature extraction. Neural networks or more specifically Convolutional Neural Networks were applied to visual tasks since the late 1980 s and were mainly dormant until the mid-2000s when better developments in computer power and algorithms were created (Rawat and Wang 2018). The ensuring paragraphs discuss some of the history of deep learning research, neural network architecture and the different applications of neural networks to healthcare and security over the recent years.

\section{Deep Neural Networks and Convolutional Neural Networks (CNN)}

Artificial deep neural networks, mostly recurrent ones, have received huge acclaim in competitions about machine learning and pattern recognition. Schmidhuber (2015) evaluated most of the work relevant to artificial deep neural networks from the past millennium. He found that the various forms of machine learning could be differentiated by the links between actions and effects as well as their depth of credit assignment paths. He reviewed unsupervised learning, reinforcement learning \& evolutionary computation, deep supervised learning, the history of backpropagation, and indirect search for short programs encoding deep and large networks.

Convolutional Neural Networks gained notoriety when they won the ImageNet Large Scale Visual Recognition Challenge in 2012(ILSVRC12). The ImageNet project is a large visual database designed for use in visual object recognition research and they held this contest challenge annual from 2010-2017. ImageNet contains more than 20,000 categories and for purposes of the challenge about 150,000 image datasets taken from web are provided to researchers for correctly classify and detect object and scenes.

Other Researchers have since 2012 paid a lot of attention to Convolutional Neural Network after it won the Image Classification Competition, ILSVRC12. The CNN success was attributed to its superior multi-scale high-level image representations compared to hand-engineering low-level features. There are some challenges with CNN such as a requirement of large amount of labeled training samples and a powerful GPU to accelerate the learning process. These requirements could limit the number of people who could utilize CNN. 
Han et al (2018) addressed one of the major problems involved with estimating millions of parameters for a deep $\mathrm{CNN}$, which requires a large number of annotated samples therefore preventing CNNs like AlexNet or ResNet to be applied to problems with limited training data by proposing a two-phase method combining CNN transfer learning and web data augmentation. This method not only reduced the requirement of large training data, it also more efficiently expanded the training dataset and reduced over-fitting on small dataset by first transferring useful feature presentation of a pre-trained network to a target task and then augmenting the original dataset with valuable Internet images for classification. Han et al (2018) interestingly applied a Bayesian optimization algorithm to hyper-parameters tuning for fine-tuning the network and applied it to six small datasets available publicly. They proposed from experiments carried that their solution applied to popular deep CNNs like ResNet can improve performance for small datasets, hence making their proposed solution available for tackling problems related to applying deep CNNs on small datasets.

\section{ResNET}

Zagoruyko and Komodakis (2016) studied the Convolutional Neural Network architecture known as ResNet blocks by decreasing depth and increasing width of residual networks and renaming it Wide Residual Networks (WRNs). They found that very deep networks were actually a weakness as it hindered learning during training or that very little learned information was shared. In other words, the ResNet style architecture was producing diminishing feature reuse because the minimal gains in accuracy came a large cost of doubling the number of layers. By widening the approach as opposed to shallow and deeper, their wider residual network (WRN) significantly improved results with 50 less layers and performing more than 2 times faster. In fact, they were able to demonstrate that a simple 16-layer-deep WRN outperforms previous datasets (CIFAR-10, CIFAR-100, SVHN, COCO, and significant improvements on ImageNet) in accuracy and efficiency.

\section{PCANet}

Chan et al (2015) proposed very simple deep learning network called PCANet for image classification (faces, digits, and texture). PCANet is described as being based on 3 data processing components; one is cascaded principal component analysis, second is binary hashing and the third is blockwise histograms. PCANet also comes in 2 variations: RandNet, where cascaded filters are chosen randomly and LDANet, where the filters are chosen more arbitrarily by way of some linear analysis. The authors tested PCANet on many different benchmark visual datasets for different tasks (face verification, face recognition, and hand-written digit recognition) and found its performance to be comparable and that PCANet can be a valuable baseline for studying advanced deep learning architectures.

\section{MCDNN}

Ciregan et al (2015) claim to have produced a deep artificial neural network architectures as being able to match human performance in certain tasks that would ordinarily require cognitive abilities. They compare their MCDNN to the network of neural layers found in the retina and visual cortex of mammals. Ciregan et al (2015) claim the ability of DNNs to match and outperform humans on certain benchmarks is attributed to the DNN's humanly comparable network of highly efficient neurons "winner-takes-all" neurons, which are then trained, unlike what we see in mammals. In addition, there is also further improvement for DNN on certain image classification benchmarks.

Deep Learning image classification has great potential in medical research and practice. Litjens et al (2017) reviewed over 300 contributions towards major deep learning concepts relevant to medical image analysis. The authors cite the rapid adoption of deep learning algorithms as the main methodology for analyzing medical images as the reason for their survey. They surveyed the use of deep learning for image classification, object detection, segmentation, registration, and other tasks. In addition, the study provides overview of deep learing use in more precise areas including neuro and digital pathology. The paper concludes with a discussion if the challenges presented by deep learning applications in the medical analysis field and a discussion on possibilities for future research.

\section{Applications of Deep Learning and CNN}

Liu et al (2018) sought to apply deep learning architecture solutions to the field of Magnetic Resonance Imaging research. They reviewed MRI-based deep learning applications such as image detection, registration, segmentation and classification. Liu et al (2018) presented an objective assessment of deep learning and discussed future trends and possibilities of deep learning solutions for MRI applications. 
Bychkov et al (2018) considered deep learning to have great promise in medical image classification. They combined a CNN with a recurrent architecture in an effort to predict colorectal cancer outcome based on images of tumor tissue samples. Their approach was unique as the directly predicted the outcome of the patient without any intermediate tissue classification. They suggest their results that an ideal deep learning procedure will yield more information from a cancer tissue than a human specialist could provide by observing the same sample.

Rakhin et al (2018) investigated the possibility of applying deep learning to assist with improving breast cancer diagnostic accuracy. They developed a computational approach based on deep Convolutional Neural Networks for classifying breast cancer histology images. The authors claim their approach outperformed other common methods in automated histopathological image classification.

Another area that has benefited from deep learning research is security enforcement and prevention. While humans are currently, still an important part of surveillance and control systems deep learning could provide great assistance. Reyani and Mahdavi (2007) set some of the framework for using neural networks for user identification. Sun et al (2014) created a framework that sought to examine high-level facial characteristics. The following year Sun et al (2015) augmented their previous framework to include deep learning which they aptly titled 'Deepid3.' Parkhi et al (2015) conducted a CNN model that tested face image and video recognition with on data parity and time. Mollahosseini, et al (2015) expanded previous studies by augmenting facial recognition to include facial expressions. Kumar et al (2017) investigated the using neural learning for smart security in the Internet of Things (IoT). Olmos et al (2018) illustrates how deep learning could be applied to surveillance systems. Using deep learning in the form of Convolutional Neural Networks, and the researchers defined a metric called Alarm Activation Time per Interval (AATpl) to assess performance of a detection model as an automatic detection system in videos. The results of their system shows promise, so much so that video quality provides little to no hindrance in the detection success rate.

\section{METHODOLOGY}

This research seeks to test a Convolutional Neural Network system with the task of identifying images with the objective of binary classification for decisions such as security access. There are two components of this section, with the first part containing a discussion on dataset collection, creation, cleaning, and model selection. The second part is the discussion of the process, system architecture, code snippets and test program results.

Before we discuss the implementation of this architecture, it is important to review what steps were required to acquire, clean and prepare the dataset. The gathered dataset consists of pictures of the Hollywood Celebrities, saved under "authorized" directory and images of other humans that are saved under the "not authorized" directory. Manually annotating image dataset is time consuming, difficult and quite expensive as a process. We leveraged on the availability of Google images to minimize the time to create the training dataset.

We searched the Google website for images using the Google Chrome browse for celebrity names, which were then classified from A to E. One of the search results appears as shown below in Figure 2. Next, we used JavaScript to gather the URLs associated which each images. Listed below are the steps that were used to capture the URLs, clean and prepare the image data sets.

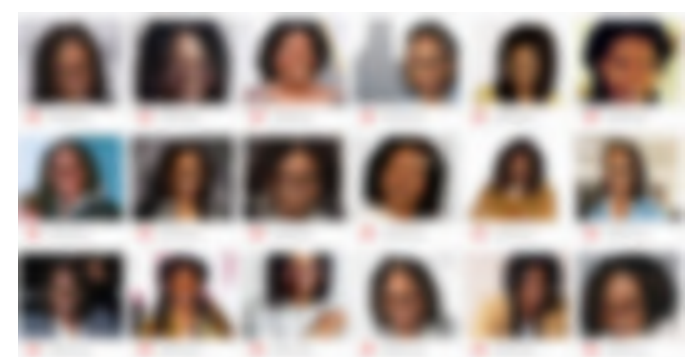

Figure 2. Image search result for Blurred Celeb E 


\section{Add JQuery JS library to the console (Figure 3)}
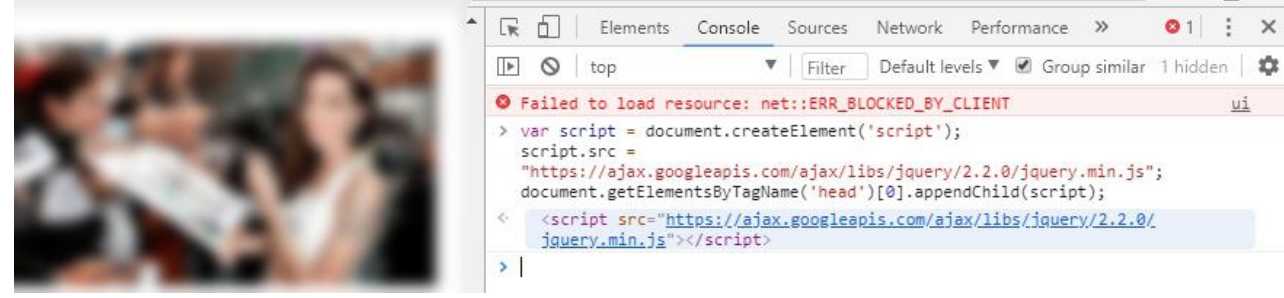

Figure 3. JavaScript Console Code

\section{Grab Image Urls}

var urls $=\$($ '.rg_di .rg_meta').map(function() $\{$ return JSON.parse(\$(this).text()).ou; $\})$;

\section{Write Image Urls to urls.txt file shown in figure $x$ below.}

var textToSave = urls.toArray().join(' 'n');

var hiddenElement $=$ document.createElement('a');

hiddenElement.href = 'data:attachment/text,' + encodeURI(textToSave);

hiddenElement.target $=$ '_blank';

hiddenElement.downloa $\overline{\bar{d}}=$ 'urls.txt';

hiddenElement.click();

4a. Data Cleaning Steps Involves ensuring only pictures of celebrities was scraped.

4b. Download the urls.txt files with OpenCV using the Python code snippets shown below

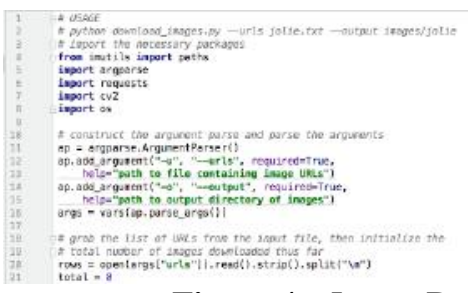

Figure 4a. Image Downloader Snippet

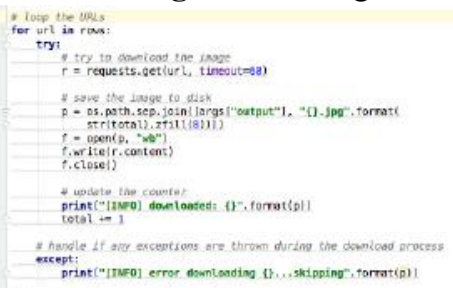

Figure 4b. Image Downloader Snippet

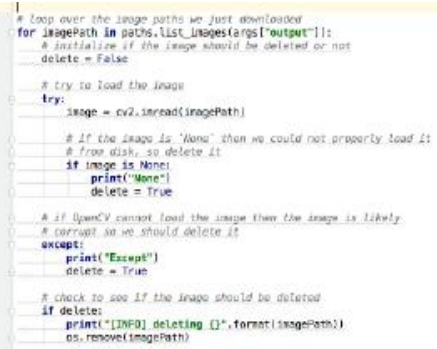

Figure 4c. Image Downloader Snippet 
Following the successful scraping of a celebrity's photo, we used the following processes for Data Preparation and Model Selection Processes shown in fig 5 and 6 respectively.

Data Preparation Process: Gather Data ---> Clean Data ---> Randomize the order ---> Visualize the Data.

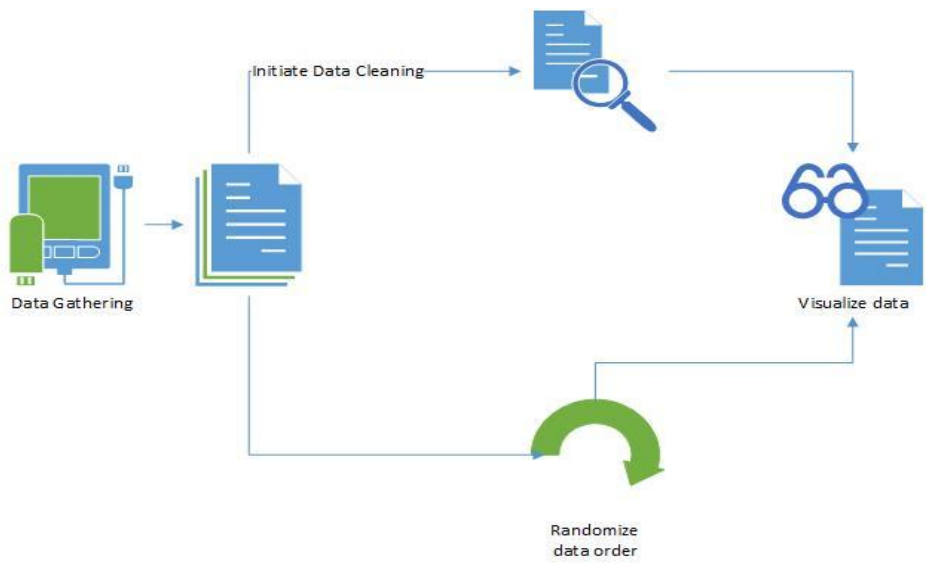

Figure 5. Data Preparation Process

Model Selection Process: Training --->Features: weights and biases ---> Evaluation (Test the model against data that hasn't been used for training with say 80:20) --->Parameter Training (Learning rate) ---->Prediction or Inference.

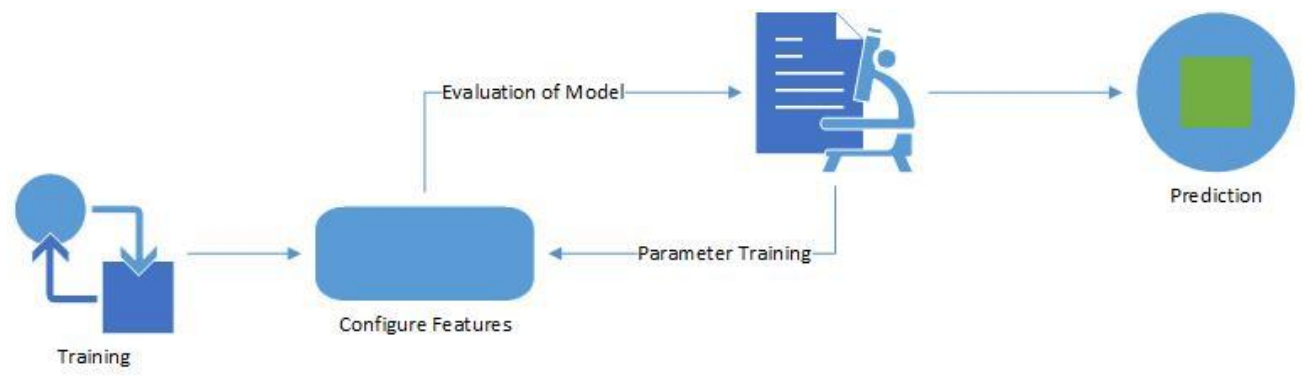

Figure 6. Model Selection Process

After the data had been collected and cleaned, the next step was training the model on image classification. Two discrete categories of "Authorized" and "Not Authorized" were maintained. We used this dataset to classify whether an image was that of a celebrity (Celeb A and Celeb E). We trained a Convolutional Neural Network based on the LeNet architecture by evaluating the "Not Authorized" model on a series of images. We sampled 704 images containing celeb A and celeb E. We then randomly sampled 704 images that do not contain either celeb A or celeb E. The next section discusses the training in more detail.

\section{TRAINING THE MODEL}

Figures $7 \mathrm{a}$ and $7 \mathrm{~b}$ are screen snips that illustrates the network training. Specifically, te train_network.py loads the dataset, preprocesses the images, instantiate our $\mathrm{CNN}$, trains the image classifier and saves plot to disk. 


\section{Issues in Information Systems}

Volume 20, Issue 3, pp. 107-116, 2019

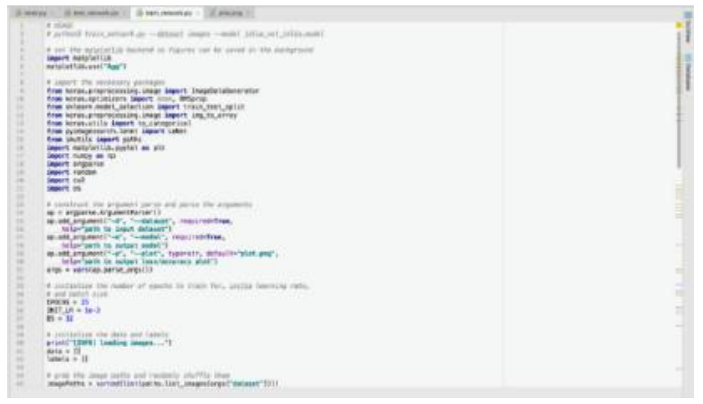

Figure 7a. Snippet of Network Trainer

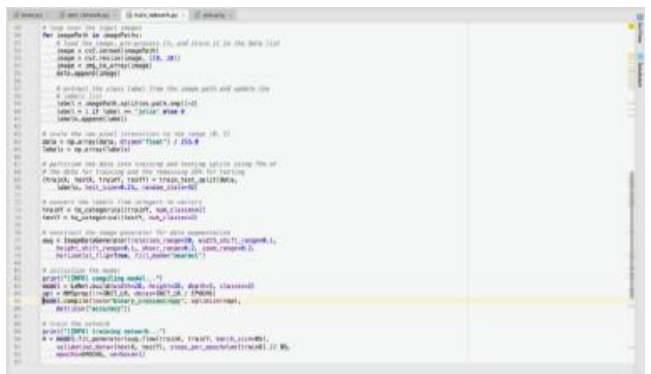

Figure 7b. Snippet of Network Trainer

The next step is consider optimizers available on keras, and then to compile our model. Optimizers like Adam, Stochastic gradient descent (SGD), AdaGrad, RMSprop, Nadam and AdaDelta were evaluated and highlights of each are discussed below.

- Adam: This is an adaptive moment estimation with relatively low memory requirements that works well with little hyperparameter tuning.

- $\quad$ SGD: This optimizer works well for shallow networks and has very slow convergence.

- AdaGrad: This optimizer is well suited for dealing with sparse data because it makes small updates for frequent parameters and big updates for infrequent parameters with a major benefit of not needing manual tuning of the learning rate. Its major disadvantage is with the always decaying and decreasing learning rate.

- $\quad$ RMSprop: This optimizer is a good choice for recurrent neural networks and its learning rate can be freely tuned.

- Nadam: This is a Nestorov Adam optimizer. Nadam is essentially Adam RMSProp with Nesterov momentum.

- $\quad$ AdadDelta: This optimizer doesn't require its learning rate to be set. It is an extension of the AdaGrad optimizer but removes its decreasing learning rate problem.

We built our LeNet model with the these optimizers and compared the results. Since this is a two-class classification problem we used a binary cross-entropy (instead of categorical since classes are not greater than 2) as our loss function. We used the following command to run the train_network.py file.

Python3 train_network.py-dataset images --model test.model

\section{EVALUATION OF CLASSIFIER}

We evaluated our model on some test images that were not part of the training and testing splits by running the test_network.py file which accepts the model and image as binary arguments. This process predicts the accuracy of the input image for both authorized and not authorized and displays the results. 


\section{RESULTS}

Evaluation results show that the network trained for 50 epochs (full forward and backward pass of dataset) achieved an accuracy of $96.7 \%$ and low loss that follows the training loss. Figure 8 illustrates that all of the models trained have accuracies above 0.96 . It was noted that the stochastic gradient descent (SGD) optimizer had the lowest value that was due to its slow convergence.
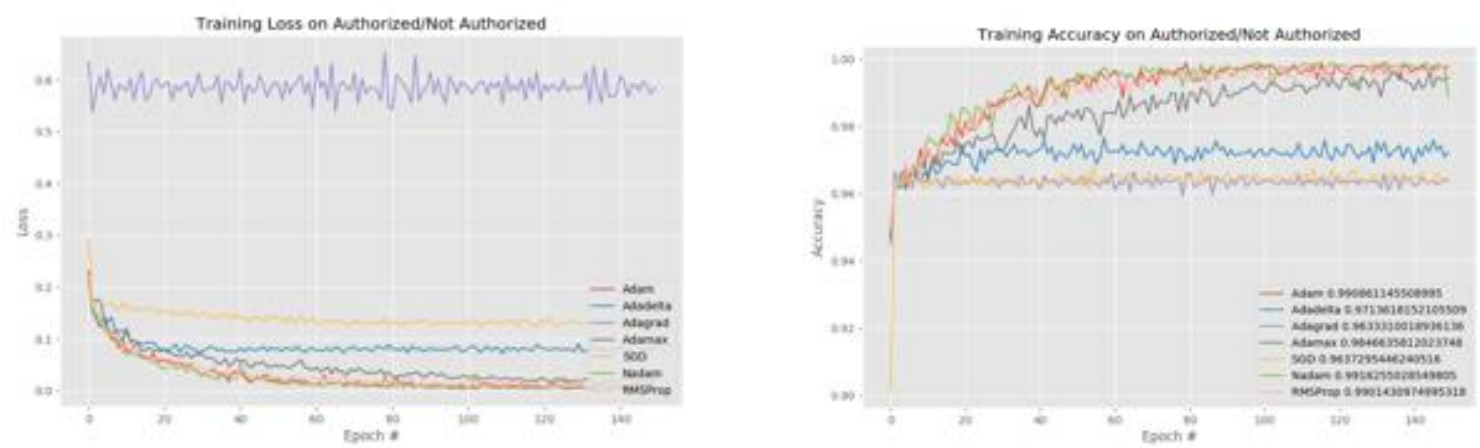

Figure 8. Showing Loss and Accuracy curves

Figure 9 shows the various results for our model evaluation on some test images. Tests show that identifying a non authorized face was done at extreme accuracy. Randomly testing sample images to test if they are authorized or not show accuracy results at between $96-99 \%$. The probability of an image properly classified was calculated to be 0.98 using the weighted average of the number tests performed.

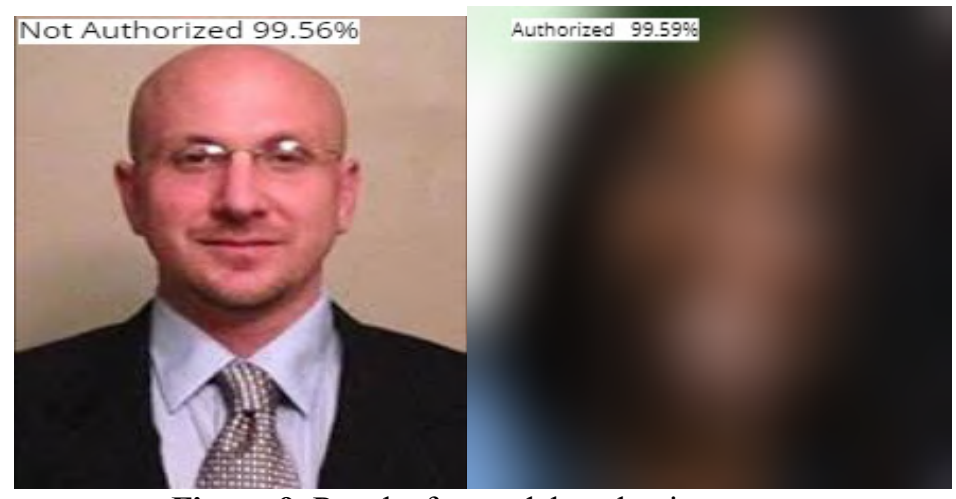

Figure 9. Results for model evaluation

\section{CONCLUSION}

The outcome of our model was successful. The results provide evidence that deep learning and convolutional neural networks performed quite well for determining authorized and unauthorized access. The success of this technology would satisfy a very important need and function of smart home security systems. For example, this program would allow for quicker identification of approaching individuals to increase the protection of the home and individuals. There were some limitations to this study, such as, this model only worked on a specific computing architecture and framework. In addition, the accuracy of our classifier was limited to a certain set of constraints. Future studies should be conducted on exploring other architectures like ResNet and GoogleNet, which usually require GPU usage on images or real-time video streams. The accuracy of our classifier could be tested by adjusting some of the constraints. Some examples of future research that could be adjusted include increasing the training set, adjusting the input shape, expanding the input width, or augmenting the number of convolutional layers has any impact or improvement. Lastly, since deep learning models are already being applied to mobile applications, it would be interesting to integrate this model to an Android or iOS application as to allow for portable and instant image identification and classification. 


\section{REFERENCES}

Bahmani, M. J. (2018). “AI vs Machine Learning vs Deep Learning” https://medium.com/datadriveninvestor/ai-vsmachine-learning-vs-deep-learning-ba3b3c58c32, 7 November 2018. Accessed 10 May 2019

Bychkov, D., Linder, N., Turkki, R., Nordling, S., Kovanen, P. E., Verrill, C., \& Lundin, J. (2018). Deep learning based tissue analysis predicts outcome in colorectal cancer. Scientific Reports, 8(1), 3395.

Chan, T. H., Jia, K., Gao, S., Lu, J., Zeng, Z., \& Ma, Y. (2015). PCANet: A simple deep learning baseline for image classification?. IEEE transactions on image processing, 24(12), 5017-5032.

Cireşan, D., Meier, U., \& Schmidhuber, J. (2012). Multi-column deep neural networks for image classification. arXiv preprint arXiv:1202.2745.

Dhande, M. (2017). What is the difference between AI and machine language and deep learning? https://www.geospatialworld.net/blogs/difference-between-ai\%EF\%BB\%BF-machine-learning-and-deeplearning/ 6 May 2017. Accessed 10 May 2019

Fernandes, E., Jun, J. \& Prakash, A. (2016). Security risks in the age of smart homes. https://theconversation.com/security-risks-in-the-age-of-smart-homes-58756, 29 May 2016. Accessed 10 May 2019.

Fernandes, E., Jung, J., \& Prakash, A. (2016). Security analysis of emerging smart home applications. IEEE symposium on security and privacy (SP) (pp. 636-654). IEEE.

Ghazi, M. M., Yanikoglu, B., \& Aptoula, E. (2017). Plant identification using deep neural networks via optimization of transfer learning parameters. Neurocomputing, 235, 228-235.

Han, D., Liu, Q., \& Fan, W. (2018). A new image classification method using CNN transfer learning and web data augmentation. Expert Systems with Applications, 95, 43-56.

Halpin, M. (2019). Trust Issues: New Survey Sheds Light on Americans’ Biggest Security Concerns.

https://www.asecurelife.com/smart-home/trust-issues-technology-survey, 22 April 2019. Accessed 10 May 2019.

He, X., \& Chua, T. S. (2017, August). Neural factorization machines for sparse predictive analytics. In Proceedings of the 40th International ACM SIGIR conference on Research and Development in Information Retrieval (pp. 355-364). ACM.

Hinton, G., Deng, L., Yu, D., Dahl, G., Mohamed, A. R., Jaitly, N., \& Sainath, T. (2012). Deep neural networks for acoustic modeling in speech recognition. IEEE Signal Processing Magazine, 29.

Hohman, F. M., Kahng, M., Pienta, R., \& Chau, D. H. (2018). Visual analytics in deep learning: An interrogative survey for the next frontiers. IEEE Transactions on Visualization and Computer Graphics.

International Energy Agency (IEA) (2013) Energy efficiency market report. International Energy Agency (IEA), Paris, France https://www.iea.org/publications/freepublications/publication/EEMR2013_free.pdf

International Energy Agency (IEA) (2016) Medium-Term Energy efficiency market report. International Energy Agency (IEA), Paris, France https://www.iea.org/eemr16/files/medium-term-energy-efficiency2016_WEB.PDF

Lawrence, C. (2017). Dojo brings critical security to smart home automation. https://readwrite.com/2017/03/27/dojo-brings-critical-security-to-smart-home-automation-dl4/, 27 March 2017. Accessed 10 May 2019.

Le, Q. V., Ranzato, M. A., Monga, R., Devin, M., Chen, K., Corrado, G. S., \& Ng, A. Y. (2011). Building high-level features using large scale unsupervised learning. arXiv preprint arXiv:1112.6209.

Liu, J., Pan, Y., Li, M., Chen, Z., Tang, L., Lu, C., \& Wang, J. (2018). Applications of deep learning to MRI images: A survey. Big Data Mining and Analytics, 1(1), 1-18.

Litjens, G., Kooi, T., Bejnordi, B. E., Setio, A. A. A., Ciompi, F., Ghafoorian, M., \& Sánchez, C. I. (2017). A survey on deep learning in medical image analysis. Medical Image Analysis, 42, 60-88. 
Long, J., Shelhamer, E., \& Darrell, T. (2015). Fully convolutional networks for semantic segmentation. In Proceedings of the IEEE conference on computer vision and pattern recognition (pp. 3431-3440).

Kumar, P.M., Ghandi, U., Varatharajan, R., Manogaran, G., Jidesh, R., \& Vadivel, T. (2017). Intelligent face recognition and navigation system using neural learning for smart security in Internet of Things. Cluster Computing, 1-12.

Mollahosseini, A., Chan, D., \& Mahoor, M. H. (2016, March). Going deeper in facial expression recognition using deep neural networks. In 2016 IEEE Winter conference on applications of computer vision (WACV) (pp. 110). IEEE

Olmos, R., Tabik, S., \& Herrera, F. (2018). Automatic handgun detection alarm in videos using deep learning. Neurocomputing, 275, 66-72.

Parkhi, O.M., Vedaldi, A., \& Zisserman, A., (2015, September) Deep Face Recognition. In bmvc 1(3), 6.

Rakhlin, A., Shvets, A., Iglovikov, V., \& Kalinin, A. A. (2018, June). Deep convolutional neural networks for breast cancer histology image analysis. In International Conference Image Analysis and Recognition (pp. 737744). Springer, Cham.

Rawat, W., \& Wang, Z. (2017). Deep convolutional neural networks for image classification: A comprehensive review. Neural Computation, 29(9), 2352-2449.

Reyhani, S.Z., and Mahdavi M., (2007) User authentication using neural networks in smart home networks. International Journal of Smart Home, 1(2), 147-154.

Schmidhuber, J. (2015). Deep learning in neural networks: An overview. Neural Networks, 61, 85-117.

Shu, X., Cai, Y., Yang, L., Zhang, L., \& Tang, J. (2017). Computational face reader based on facial attribute estimation. Neurocomputing, 236, 153-163.

Sun, Y., Wang, X., \& Tang, X. (2014) Deep learning face representation from predicting 10,000 classes. In Proceedings of the IEEE conference on computer vision and pattern recognition (pp.1891-1898).

Sun, Y., Wang, X., \& Tang, X. (2015) Deepid3: Face recognition with very deep neural networks. arXiv preprint arXiv:1502.00873.

Skymind (2019) “A.I. Wiki” https://skymind.ai/wiki/ai-vs-machine-learning-vs-deep-learning Accessed 12 July 2019

https://www.spglobal.com/marketintelligence/en/news-insights/blog/smart-homes-in-the-u-s-becoming-morecommon-but-still-face-challenges Accessed 12 July 2019

Statista (2018) Smart Home Revenue Growth and Prediction, https://www.statista.com/outlook/279/109/smarthome/united-states, Retrieved 10 May 2019

Taigman, Y., Yang, M., Ranzato, M. A., \& Wolf, L. (2014). Deepface: Closing the gap to human-level performance in face verification. In Proceedings of the IEEE conference on computer vision and pattern recognition (pp. 1701-1708).

Van Alphen, R. (2019) https://schoolofdisruption.com/exponential-technologies/artificial-intelligence/ Accessed 10 May 2019

Yu, W., Yang, K., Yao, H., Sun, X., \& Xu, P. (2017). Exploiting the complementary strengths of multi-layer CNN features for image retrieval. Neurocomputing, 237, 235-241.

Zagoruyko, S., \& Komodakis, N. (2016). Wide residual networks. arXiv preprint arXiv:1605.07146.code at https://github.com/szagoruyko/wide-residual-networks 